\section{Use of a microprocessor and a digital computer in the analysis of rodent mating behavior}

\author{
JOHN R. LEU and SHELTON E. HENDRICKS \\ University of Nebraska at Omaha, Omaha, Nebraska 68182
}

Accurate recording of the sequence and timing of behaviors is a problem common to many areas of research. Our laboratory investigations are often concerned with the character and temporal patterning of sexual responses of various species of rodents. We have devised a system utilizing a Datamyte 900 (Electro/General Corporation, 14960 Industrial Road, Minnetonka, Minnesota 55343) and an interactive computer system (Control Data Corporation Cyber 172, operating under the NOS timesharing system), which allows for relative ease of data collection, minimization of errors, and provision of detailed summary information within a matter of minutes. The raw data are permanently stored in computer-readable format, facilitating future analyses. Although the program was developed for the specific purpose of summarizing the male sexual behavior of rats, additional programs summarizing other aspects of reproductive behavior of rodents have been developed. Programs summarizing female sexual behavior of rats and simultaneous male and female behavior of mice and additional programs describing male sexual behavior have been written. These programs can be adopted to the analysis of any behavior that can be discretely categorized.

The Datamyte is an electronic device that allows recording, storage, and transmission of response codes and their elapsed times in any of several formats. The device can be either battery powered or operated from line voltage, and it is easily portable. Information is stored electronically and is subsequently transmitted directly to a computer for permanent storage and analysis.

Input. The basic observational conditions and scoring procedures have been described elsewhere (Hendricks \& Scheetz, 1973). Briefly, the male reproductive behavior of rats is categorized as to the occurrence and timing of mounts, intromissions, and ejaculations. As many as eight animals may be observed simultaneously, each in a separate observation chamber. Responses are coded with the Datamyte set in an input mode described as two-digit autotime. In this mode, for each behavioral event, the observer first enters the "box number" (the number of the observation chamber, 1-8) and next enters the response code ( 1 for a mount, 2 for an intromission, 3 for an ejaculation). The Datamyte then automatically stores a complete record, which includes the box number and response code along with the elapsed time in hundredths of a minute.
Following data collection, the data are transmitted to the computer using an output mode that transmits each entry with a line number, two entry codes, elapsed time, carriage return, and line feed. A permanent file containing the raw data records for as many as eight animals is now available for analysis.

Output. To summarize the data meaningfully, a program was written to do three things: First, a separate raw data record is provided for each animal, with elapsed time given in seconds. Next, a summary table is created, giving the most commonly used measures describing male sexual behavior. The final output is a response histogram giving a graphic representation of the responses over time. The program is interactive and provides some help and prompting when necessary. All animals or only specified animals can be summarized. If desired, any of the three types of output can be suppressed.

Restrictions and Capacity. The use of the Datamyte in conjunction with this program has provided us convenient, accurate recording of behavioral data and very rapid, detailed summaries. The data are readily available for subsequent analyses. However, there are certain limitations. As written, the program accommodates tests of up to $65 \mathrm{~min}$ in length. Longer tests can be recorded and summarized by placing an end-of-record marker each hour and summarizing each hour as a separate test. The Datamyte has a large but finite capacity (we have yet to fill it with an afternoon's testing). On some occasions, the time limit of the battery packs could be a consideration, but this is no problem if line voltage is available. Data need not be transmitted immediately and can be stored indefinitely if auxiliary power is provided. This program can be expanded as desired to include other categories of behavior. We are currently modifying this program to accommodate and summarize mountbouts, as described by Sachs and Barfield (1972). Other behaviors that might be included are grooming, vocalization, fighting, and escape.

Availability. The use of this program is not restricted. Documented listings of this and other related programs can be obtained without charge from the authors. The authors acknowledge the assistance of the staff of the University of Nebraska computer network.

\section{REFERENCES}

Hendricks, S. E., \& Scheetz, H. A. Interaction of hypothalamic structures in the mediation of male sexual behavior. Physiology \& Behavior, 1973, 10, 711-716.

SAchs, B. D., \& Barfield, R. J. Temporal patterning of male sexual behavior in the male rat. Journal of Comparative and Physiological Psychology, 1970, 73, 359-364.

(Accepted for publication May 23, 1980.) 